\title{
Comparison of early and on-demand maternal feeding after Caesarean delivery: a prospective randomised trial
}

\author{
Esra Ozbasli ${ }^{1}$, MD, Ozguc $\underline{\text { Takmaz }}^{1}$, MD, Faruk Suat Dede ${ }^{1}$, MD, Mete Gungor ${ }^{1}$, MD
}

INTRODUCTION This study aimed to compare early and on-demand maternal feeding after Caesarean delivery in terms of gastrointestinal complaints and patient satisfaction.

METHODS A total of 262 women with uncomplicated singleton term pregnancies who underwent a Caesarean section under regional anaesthesia were randomised to a soft food diet served at Postoperative Hour 2 (early feeding group) or eating whenever they wanted to upon return to the ward (on-demand group). Patient satisfaction scores at the time of discharge and gastrointestinal complaints were compared.

RESULTS The fed-early group comprised 133 (50.8\%) women and the on-demand group comprised 129 (49.2\%) women. Major characteristics and surgical procedures were comparable between the two groups. No significant between-group differences in demographic criteria or surgical procedures were evident $(p>0.05)$. The mean time to the first feeding was $120.00 \pm 00.00$ minutes for the early feeding group as compared to $236.59 \pm 107.74$ minutes for the on-demand feeding group $(p=0.001)$. Satisfaction levels did not differ significantly between the two groups $(p=0.366)$. Duration to first breastfeeding, analgesia on the ward, passage of flatus, defecation, mobilisation and urination after catheter removal did not differ significantly between the two groups $(p>0.05)$.

CONCLUSION Early initiation of solid food in low-risk women after Caesarean delivery under regional anaesthesia was associated with high satisfaction and did not increase gastrointestinal complaints. We suggest having flexibility in terms of postoperative feeding time. This may shorten hospitalisation time and reduce hospitalisation costs.

Keywords: Caesarean delivery, gastrointestinal complaints, patient satisfaction, postoperative feeding, prospective randomised trial

\section{INTRODUCTION}

Caesarean delivery rates are increasing worldwide. ${ }^{(1)}$ As a result, postoperative care is gaining momentum in terms of shortening hospitalisation time, reducing the rate of hospital infection and financial costs, and increasing patient satisfaction. Postoperative pain and gastrointestinal complaints are the most common complaints after a Caesarean section. ${ }^{(2)}$ Optimisation of postoperative feeding time may increase patient satisfaction and reduce postoperative complaints. ${ }^{(3,4)}$ However, no standard postoperative feeding time is available; the timing varies among clinics, ${ }^{(5,6)}$ ranging from the second postoperative hour to the first passage of flatus or a bowel movement. Gastrointestinal complications, such as vomiting and ileus, and the need for emergency re-interventions are of concern when determining feeding time.

In this study, we compared early and on-demand maternal feeding after Caesarean delivery in terms of gastrointestinal complaints and patient satisfaction.

\section{METHODS}

We recruited a total of 262 women with uncomplicated singleton term pregnancies who underwent Caesarean section under regional anaesthesia between November 2017 and March 2018. This study was registered at ClinicalTrials.gov (no. NCT03349151, https://clinicaltrials.gov/ct2/show/NCT03349151) and was approved by the Medical Ethics Committee of Acibadem MAA University, Istanbul, Turkey (ATADEK ID no. 2017-4/13).

Women with uncomplicated singleton term pregnancies who underwent an uncomplicated elective Caesarean section performed under regional anaesthesia were included in our study. Exclusion criteria were general anaesthesia, history of bowel surgery, diagnosis of a fetal anomaly, history of maternal disease (cardiovascular disease, chronic hypertension, thromboembolic disorders, gastrointestinal and hepatic disorders, or diabetes mellitus), and intraoperative or immediate postoperative complications. All patients provided written informed consent. Using a computer-generated sequence concealed from the obstetrician and patients, the patients were randomised to a soft food diet (honey, skimmed cheese, soup, mashed potatoes, pudding and grissini) served during Postoperative Hour 2 or whenever they wanted to eat after returning to the ward. Opaque, sealed envelopes with the selected regimen were attached to the files of the patients included in the study. The nurse opened the envelopes at the end of the surgery. The nurse who questioned the patients and filled in the forms was blinded to the study.

All enrolled women received 10.0-12.5 mg of bupivacaine hydrochloride and $50 \mu \mathrm{g}$ of fentanyl or 10-12.5 mg of bupivacaine hydrochloride, $50 \mu \mathrm{g}$ of morphine and $10 \mu \mathrm{g}$ of fentanyl in the operating room. All patients had a Pfannenstiel incision for a laparotomy and a transverse incision at the lower uterine segment for a hysterotomy. Cefazolin sodium (2 g) was given to all patients for prophylaxis just before the skin incision. A uterine incision with a continuous single-layer closure was performed in all patients with Number 1 polyglactin 910 sutures. The rectouterine pouch and paracolic gutters were cleaned before closing the parietal peritoneum. Peritoneal closure was performed with $2 / 0$ poly(glycolide-co-lactide).

${ }^{1}$ Acibadem Mehmet Ali Aydinlar University, Acibadem Maslak University Hospital, Obstetrics and Gynecology Department, İstanbul, Turkey

Correspondence: Dr Esra Ozbasli, Assistant Professor, Acibadem Mehmet Ali Aydinlar University, Acibadem Maslak University Hospital, Obstetrics and Gynecology Department, Darüşşafaka, Büyükdere Cad. No. 40, 34457 Sarıyer/istanbul, Turkey. esraizmit@gmail.com 
The primary outcome was patient satisfaction measured using a visual analogue scale (VAS) on the second day after surgery, before the patients were discharged. The VAS is a $100-\mathrm{mm}$ long scale with descriptions at both ends, where $0=$ lack of satisfaction about the timing of the meal after surgery and $100=$ full satisfaction about the timing of the meal after surgery. A statement was provided to explain the intended measure. Secondary outcomes were: (a) time to first postoperative passage of flatus and defecation; (b) the existence of postoperative gastrointestinal complaints such as nausea, vomiting or abdominal distention; (c) and the use of postoperative pain medication. The VAS score, time to start first and second meals, amount of food consumed at the first meal, nausea, vomiting 30 minutes after first feeding, need for opiate analgesics, need for nonsteroidal antiinflammatory drugs, first analgesia on the ward, time to first passage of flatus, time to first defaecation, time to first mobilisation, and first urination after catheter removal were recorded postoperatively.

The sample size was calculated using G*Power 3 (HeinrichHeine-Universität Düsseldorf, Düsseldorf, Germany). Based on a previous study, the required sample size was 86 patients in each group to achieve $80 \%$ power for detecting a 0.43 effect size at a 0.05 significance level.

The R-3.4.3 programme ( $\mathrm{R}$ Core Team, R Foundation for Statistical Computing, Vienna, Austria) was used for the statistical analysis. The normality of the variables was assessed using the Shapiro-Wilk test. Descriptive statistical methods (mean, standard deviation and frequency) were used to evaluate the data. Student's $t$-test was used to compare normally distributed quantitative data, and the Mann-Whitney $U$-test was used for non-normally distributed quantitative variables. The chi-square test with continuity (Yates) correction was used to evaluate qualitative variables. A p-value $<0.05$ was considered statistically significant.

\section{RESULTS}

A total of 328 women underwent Caesarean sections between November 2017 and March 2018. Of these, 58 women were excluded from the study. A total of 270 women were recruited and randomised to treatment: 135 women were allocated to the early intervention group, of whom 133 completed the study; another 135 women were allocated to the on-demand group, of whom 129 completed the study. The remaining 262 women were analysed, with 133 (50.8\%) in the fed-early group and $129(49.2 \%)$ in the on-demand group (Fig. 1). The major characteristics and surgical procedures of the women and the study groups are shown in Table I.

The mean age of the participants ranged from 21.00 to 44.00 (mean $33.00 \pm 4.10$ ) years and the body mass index ranged from 17.30 to 46.30 (mean $28.21 \pm 4.07$ ) kg/m². A total of $174(66.4 \%$ ) participants had nulliparity. Gestational age at delivery ranged from 37.0 to 41.2 (median 39.0) weeks. 81 (30.9\%) participants had undergone a repeat Caesarean section. Combined spinal-epidural analgesia was used for 185 (70.6\%) participants. Morphine was used as regional analgesia in $112(42.7 \%)$ participants. The duration of surgery ranged from 30 to 120 (median 60) minutes. No significant between-group differences in characteristics or surgical procedure variables were evident (Table I).

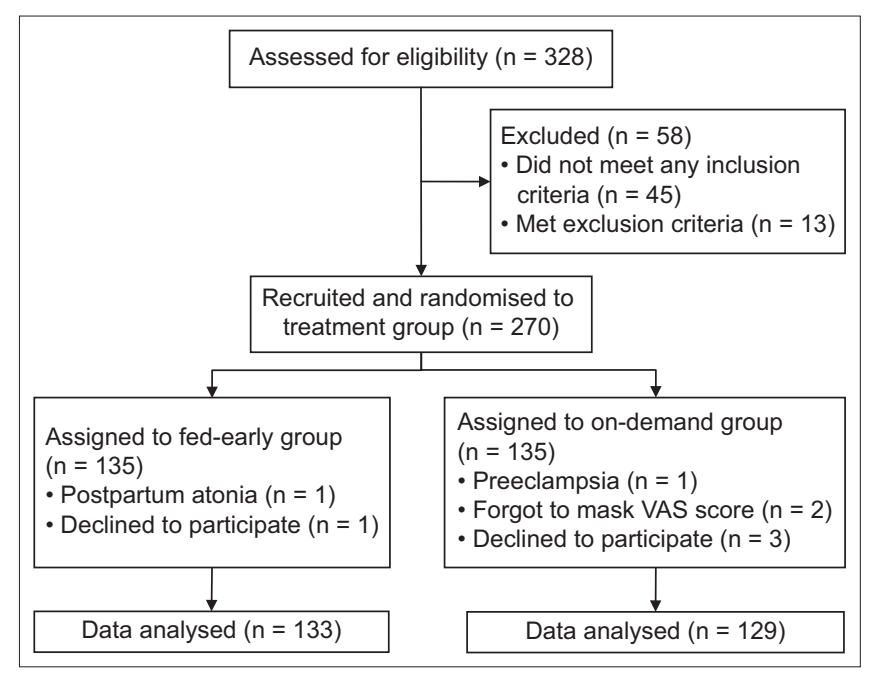

Fig. 1 Patient flowchart shows the process from recruitment to study completion. VAS: visual analogue scale

Results for the early and on-demand feeding groups are shown in Table II. The mean time to first feeding was $120.00 \pm 00.00$ minutes for the early feeding group, compared to $236.59 \pm 107.74$ minutes for the on-demand feeding group $(p=0.001)$. The mean time to the second meal was $409.64 \pm 154.96$ minutes for the early feeding group, compared to $553.60 \pm 194.94$ minutes for the on-demand feeding group $(p=0.001)$. Satisfaction tended to be higher in the early feeding group than in the on-demand group $(p=0.366)$. Duration to first breastfeeding, analgesia on the ward, passage of flatus, defecation, mobilisation, and urination after catheter removal did not differ significantly between the study groups ( $p \geq 0.05$ ).

\section{DISCUSSION}

Our results showed that early feeding is well tolerated in women undergoing uncomplicated Caesarean delivery under regional anaesthesia, which is consistent with most studies in the literature. ${ }^{(3,4,7,8)}$ Our primary outcome, patient satisfaction, showed similar scores between the early feeding and on-demand feeding groups. This finding is compatible with those of previous studies. ${ }^{(3,4)}$ Our secondary outcome measures were the times to first passage of flatus and defecation, nausea and vomiting 30 minutes after first feeding, and the need for pain medication.

In meta-analyses reported in 2013 and 2015, early regular feeding was started 6-8 hours after surgery. ${ }^{(9,10)}$ In our study, a regular diet was started two hours after surgery in the early feeding group. A 2016 study in which a solid food regimen was started as early as 106 minutes, as compared to 165 minutes, reported timing and outcomes that were compatible with those of our study. ${ }^{(4)}$

Early passage of flatus has a significant role in postoperative pain control. The times to first passage of flatus and defecation were similar for both groups in our study. In a meta-analysis by Guo et al, it was found that early feeding was associated with early first flatus and early defecation. However, early feeding groups had their first meal within the first 12 hours postoperatively. In our study, all patients in both groups had their first feeding within six hours after their Caesarean section; thus, our results do not contradict the literature. ${ }^{(10)}$ The need for opiate analgesics was 
Table I. Demographic features and surgical procedures of the two groups.

\begin{tabular}{|c|c|c|c|c|}
\hline \multirow[t]{2}{*}{ Characteristic } & \multicolumn{3}{|c|}{ No. (\%) } & \multirow[t]{2}{*}{ p-value } \\
\hline & $\begin{array}{l}\text { Early feeding } \\
(n=133)\end{array}$ & $\begin{array}{l}\text { On-demand feeding } \\
(n=129)\end{array}$ & $\begin{array}{l}\text { Total } \\
(n=262)\end{array}$ & \\
\hline $\operatorname{Age}^{*}(y r)$ & $33.20 \pm 3.91$ & $32.78 \pm 4.28$ & $33.00 \pm 4.10$ & $0.408^{\ddagger}$ \\
\hline Body mass index ${ }^{*}\left(\mathrm{~kg} / \mathrm{m}^{2}\right)$ & $27.95 \pm 3.80$ & $28.48 \pm 4.33$ & $28.21 \pm 4.07$ & $0.296^{\ddagger}$ \\
\hline Nulliparity & $92(69.2)$ & $82(63.6)$ & $174(66.4)$ & $0.337^{\S}$ \\
\hline Gestational age at delivery ${ }^{\dagger}(w k)$ & $39(28.2-39.4)$ & $39(38.4-39.2)$ & $39(38.3-39.3)$ & $0.273^{\natural}$ \\
\hline Indication for Caesarean section & & & & $0.333^{\S}$ \\
\hline Repeat Caesarean section & $37(27.8)$ & $44(34.1)$ & $81(30.9)$ & \\
\hline Malpresentation & $11(8.3)$ & $8(6.2)$ & $19(7.3)$ & \\
\hline Macrosomia & $5(3.8)$ & $5(3.9)$ & $10(3.8)$ & \\
\hline Failure to progress & $20(15.0)$ & $8(6.2)$ & $28(10.7)$ & \\
\hline Presumed fetal compromise & $4(3.0)$ & $3(2.3)$ & $7(2.7)$ & \\
\hline Performed on request & $5(3.8)$ & $9(7.0)$ & $14(5.3)$ & \\
\hline Cephalopelvic disproportion & $33(24.8)$ & $30(23.3)$ & $63(24.0)$ & \\
\hline Others & $18(13.5)$ & $22(17.1)$ & $40(15.3)$ & \\
\hline RA technique & & & & $0.168^{\varsigma}$ \\
\hline Combined spinal-epidural & $99(74.4)$ & $86(66.7)$ & $185(70.6)$ & \\
\hline Epidural & $34(35.6)$ & $43(33.3)$ & $77(29.4)$ & \\
\hline Morphine usage during RA & $53(39.8)$ & $59(45.7)$ & $112(42.7)$ & $0.336^{\S}$ \\
\hline Surgery duration ${ }^{\dagger}(\mathrm{min})$ & $60(55-70)$ & $60(57.5-75)$ & $60(55-70)$ & $0.063^{\natural}$ \\
\hline
\end{tabular}

*Data presented as mean \pm standard deviation, tmedian (25th-75th percentile). Calculated using $¥$ Student's $t$-test, §chi-square test, $\uparrow$ Mann-Whitney $U$ test. RA: regional analgesia

Table II. Results for the early and on-demand feeding groups.

\begin{tabular}{|c|c|c|c|}
\hline \multirow[t]{2}{*}{ Parameter } & \multicolumn{2}{|c|}{ Mean \pm SD (median) } & \multirow[t]{2}{*}{ p-value } \\
\hline & $\begin{array}{l}\text { Early feeding } \\
(n=133)\end{array}$ & $\begin{array}{l}\text { On-demand feeding } \\
(n=129)\end{array}$ & \\
\hline Satisfaction level (mm) & $92.30 \pm 10.66(97.00)$ & $89.65 \pm 15.79(96.00)$ & $0.366^{\dagger}$ \\
\hline Time to first feeding (min) & $120.00 \pm 0.00(120.00)$ & $236.59 \pm 107.74(210.00)$ & $0.001^{1, \neq}$ \\
\hline Food amount consumed at first feeding* & & & $0.204^{\S}$ \\
\hline Small amount & $10(7.5)$ & $4(3.1)$ & \\
\hline Moderate & $28(21.1)$ & $34(26.4)$ & \\
\hline All/almost all & $95(71.4)$ & $91(70.5)$ & \\
\hline Time to second meal (min) & $409.64 \pm 154.96(383.50)$ & $553.60 \pm 194.94(530.00)$ & $0.001^{t, \neq}$ \\
\hline \multicolumn{4}{|l|}{ Outcomes $\mathbf{3 0}$ min after first feeding* } \\
\hline Nausea & $7(5.3)$ & $11(8.5)$ & $0.424^{\S}$ \\
\hline Vomiting & $6(4.5)$ & $5(3.9)$ & $1.000^{\S}$ \\
\hline Need for opiate analgesics* & $15(11.3)$ & $18(14.0)$ & $0.641^{\S}$ \\
\hline Need for NSAID* & $102(76.7)$ & $91(70.5)$ & $0.259^{\S}$ \\
\hline First breastfeeding (min) & $65.69 \pm 44.15(60.00)$ & $67.87 \pm 33.73(60.00)$ & $0.378^{\dagger}$ \\
\hline Need for analgesia* & $53(39.8)$ & $59(45.7)$ & $0.336^{\S}$ \\
\hline First analgesia on the ward (min) & $173.86 \pm 143.8(135.00)$ & $180.23 \pm 141.5(150.00)$ & $0.581^{\dagger}$ \\
\hline Time to first passage of flatus (hr) & $17.32 \pm 8.19(16.92)$ & $18.73 \pm 9.51(17.50)$ & $0.288^{\dagger}$ \\
\hline Time to first defecation (hr) & $32.26 \pm 9.55(31.50)$ & $33.57 \pm 11.73(33.96)$ & $0.436^{+}$ \\
\hline Time to discharge from hospital (hr) & $48.5 \pm 3.5(48.00)$ & $49.4 \pm 5.8(48.00)$ & $0.11^{+}$ \\
\hline Time to first mobilisation (hr) & $5.69 \pm 1.20(5.83)$ & $5.95 \pm 1.45(6.00)$ & $0.200^{+}$ \\
\hline $\begin{array}{l}\text { Time to first urination after catheter } \\
\text { removal (hr) }\end{array}$ & $3.14 \pm 1.61(3.00)$ & $3.44 \pm 2.25(3.00)$ & $0.561^{\dagger}$ \\
\hline
\end{tabular}

*Data presented as no. (\%). †Calculated using Mann-Whitney $U$ test. $\neq \mathrm{p}<0.05$ considered statistically significant. §Calculated using chi-square test and continuity correction. NSAID: nonsteroidal anti-inflammatory drug; SD: standard deviation 
also comparable between our study groups. However, a trend of a decreased need for opiate analgesics was observed in the early feeding group. As administrating opiate analgesics depresses gastrointestinal motility, ${ }^{(11)}$ we think that having comparable gastrointestinal outcomes among our study groups may depend on the need for similar opiate analgesics administration.

Early mobilisation and early catheter removal are important components of enhanced recovery after surgery. ${ }^{(12)}$ In our study, although time to first mobilisation tended to be earlier in the early feeding group, this did not reach statistical significance. Many studies ${ }^{(3,4,7)}$ have investigated the issue of postoperative urinary catheter removal and time to hospital discharge. We did not include time to postoperative urinary catheter removal in our study, as in our hospital, we routinely remove the catheter 24 hours after surgery. Time to hospital discharge was similar between the early and on-demand feeding groups in the present study. One of the reasons for the similar discharge time might be the routine discharge protocol of our hospital. In our clinic, patients who undergo Caesarean section are discharged on the second postoperative day unless there is a complication or the patient prefers to stay longer. In addition, no significant difference in the time to first mobilisation was observed between the groups owing to our department's protocol of mobilisation at six hours postoperatively. In Guo et al's meta-analysis, the times to first mobilisation, removal of the urinary catheter and hospital discharge were shorter in the early feeding group. ${ }^{(10)}$

One of the main concerns associated with Caesarean deliveries is the timing of first breastfeeding after the surgery. The first breastfeeding time did not differ between the early feeding and ondemand feeding groups in our study. However, in a randomised controlled trial by Teoh et al, the early feeding group started first breastfeeding earlier than the control group. ${ }^{(13)}$ This difference may be attributed to varying definitions for early feeding time in the studies. In addition, time to first urination after removal of the catheter in our study groups was similar to that in Tan et al's randomised trial. ${ }^{(4)}$

Our primary and secondary outcome measures tended to be better in the early feeding group, but there was no significant difference between the groups. This similarity might be attributable to the fact that the first feeding interval between the trial arms was much shorter than the intervals reported in previous studies. Nausea was more frequent 30 minutes after first feeding in the on-demand feeding group $(8.5 \%)$ than in the early feeding group (5.3\%). However, the difference was not statistically significant. This finding is compatible with that of a 2013 meta-analysis. ${ }^{(9)}$ The incidence of vomiting was similar between our study groups, which is similar to that in a randomised trial in the study by Tan et al. ${ }^{(4)}$ Ileus or abdominal distention is a concern in starting an early solid food regimen after a surgery, but neither was observed in either group in our study.

The present study showed that early feeding and on-demand feeding after Caesarean section have similar outcomes in terms of postoperative patient satisfaction, nausea, vomiting, need for analgesics (opiate or non-opiate), time to first breastfeeding, mobilisation, flatus, defection and hospital discharge. On-demand feeding protocols may be difficult to implement, as one of the serving staff has to be instantly ready for a demand. However, patients probably prefer on-demand feeding when compared to eating according to hospital protocols.

In conclusion, early feeding and on-demand feeding are tolerable approaches after a Caesarean section, and these approaches have similar patient satisfaction levels. Thus, both approaches can be implemented in clinical practice according to physicians' preferences. Nevertheless, on-demand feeding may be superior, as it allows patients to make their own choice during the postoperative period.

\section{REFERENCES}

1. Delbaere I, Cammu H, Martens E, et al. Limiting the caesarean section rate in low risk pregnancies is key to lowering the trend of increased abdominal deliveries: an observational study. BMC Pregnancy Childbirth 2012; 12:3.

2. Moss G, Regal ME, Lichtig L. Reducing postoperative pain, narcotics, and length of hospitalization. Surgery 1986; 99:206-10.

3. Izbizky GH, Minig L, Sebastiani MA, Otaño L. The effect of early versus delayed postcaesarean feeding on women's satisfaction: a randomised controlled trial. BJOG 2008; 115:332-8.

4. Tan PC, Alzergany MM, Adlan AS, Noor Azmi MA, Omar SZ. Immediate compared with on-demand maternal full feeding after planned caesarean delivery: a randomised trial. BJOG 2017; 124:123-31.

5. Mangesi L, Hofmeyr GJ. Early compared with delayed oral fluids and food after caesarean section. Cochrane Database Syst Rev 2002; (3):CD003516.

6. Masood SN, Masood Y, Naim U, Masood MF. A randomized comparative trial of early initiation of oral maternal feeding versus conventional oral feeding after cesarean delivery. Int J Gynaecol Obstet 2014; 126:115-9.

7. Barat S, Esmaeilzadeh S, Golsorkhtabaramiri M, Khafri S, Recabdarkolaee MM. Women's satisfaction in early versus delayed postcaesarean feeding: a one-blind randomized controlled trial study. Caspian J Intern Med 2015; 6:67-71.

8. Mulayim B, Celik NY, Kaya S, Yanik FF. Early oral hydration after cesarean delivery performed under regional anesthesia. Int J Gynaecol Obstet 2008; 101:273-6.

9. Hsu YY, Hung HY, Chang SC, Chang YJ. Early oral intake and gastrointestinal function after cesarean delivery: a systematic review and meta-analysis. Obstet Gynecol 2013; 121:1327-34.

10. Guo J, Long S, Li H, et al. Early versus delayed oral feeding for patients after cesarean. Int J Gynaecol Obstet 2015; 128:100-5.

11. Kaufman PN, Krevsky B, Malmud LS, et al. Role of opiate receptors in the regulation of colonic transit. Gastroenterology 1988; 94:1351-6.

12. Varadhan $\mathrm{KK}, \mathrm{Neal} \mathrm{KR}$, Dejong $\mathrm{CH}$, et al. The enhanced recovery after surgery (ERAS) pathway for patients undergoing major elective open colorectal surgery: a meta-analysis of randomized controlled trials. Clin Nutr 2010; 29:434-40.

13. Teoh WHL, Shah MK, Mah CL. A randomised controlled trial on beneficial effects of early feeding post-Caesarean delivery under regional anaesthesia. Singapore Med J 2007; 48:152-7.

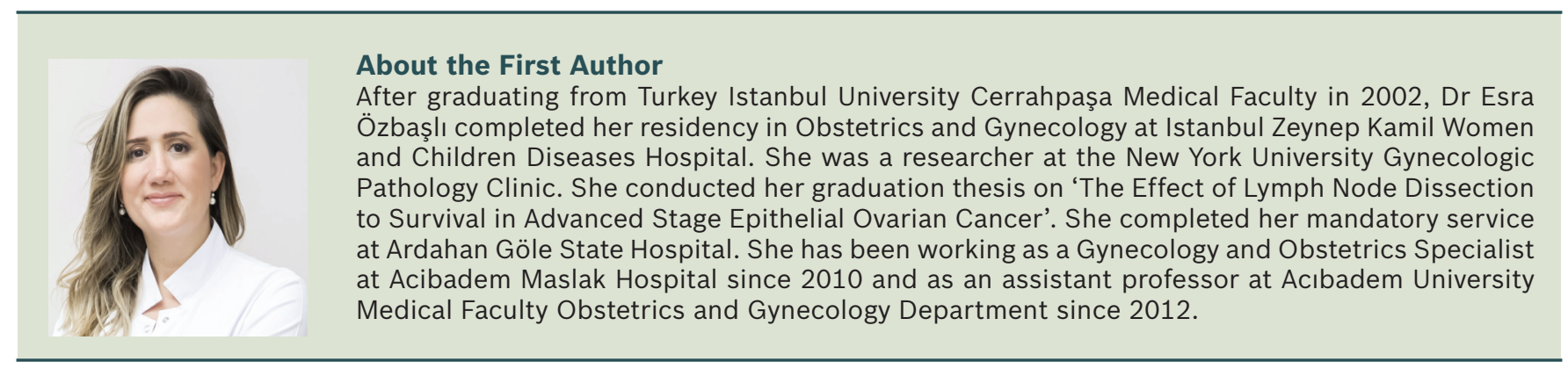

